\title{
Symbiotic algae-bacteria dressing for producing hydrogen to accelerate diabetic wound healing
}

Huanhuan Chen ${ }^{1 \dagger}$, Yunfei Guo ${ }^{1 \dagger}$, Zhewei Zhang ${ }^{1}$, Wenxuan $\mathrm{Mao}^{1}$, Chenying Shen ${ }^{1}$, Wei Xiong ${ }^{2}$, Yingfang $\mathrm{Yao}^{2}$, Xiaozhi Zhao ${ }^{3}$, Yiqiao $\mathrm{Hu}^{1,4}$, Zhigang Zou², Jinhui $\mathrm{Wu}^{1,4,5 *}$

\section{Affiliations:}

${ }^{1}$ State Key Laboratory of Pharmaceutical Biotechnology, Medical School \& School of Life Sciences, Nanjing University, Nanjing 210093, China

${ }^{2}$ Eco-materials and Renewable Energy Research Center (ERERC), College of Engineering and Applied Sciences, Nanjing University, Nanjing 210093, China

${ }^{3}$ Department of Medical Laboratory, Nanjing Drum Tower Hospital Affiliated to Medical College, Nanjing University, Nanjing, 210008, China

${ }^{4}$ Jiangsu Provincial Key Laboratory for Nano Technology, Nanjing University, Nanjing 210093, China

${ }^{5}$ Chemistry and Biomedicine Innovation Center, Nanjing University, Nanjing 210023, China

These authors contributed equally: Huanhuan Chen, Yunfei Guo

${ }^{*}$ Corresponding author. Email: wuj@nju.edu.cn (J.W.) 


\section{Experimental Section}

\section{Oxygen consumption of Bac-Chl solution}

The $\mathrm{O}_{2}$ concentration measurements were acquired by the oxygen electrodes $(\mathrm{OX}-\mathrm{N}-$ 904151, Unisense). The chlorella and Bacillus solutions were collected and centrifuged at $3500 \mathrm{rpm}$ and $6000 \mathrm{rpm}$ for 5 minutes, respectively. Remove the supernatant, and then repeatedly wash with PBS three times, and then resuspend the chlorella in PBS and bacteria in LB medium for later use. Add bacteria with different concentrations $\left(8 \times 10^{8}, 9 \times 10^{8}, 1 \times 10^{9}, 1.2 \times 10^{9}\right.$, and $1.5 \times 10^{9}$ cells $\left./ \mathrm{mL}\right)$ to chlorella solution $\left(1 \times 10^{8}\right.$

cells $/ \mathrm{mL}$ ) to get Bac-Chl solution with different ratios of 1:8, 1:9, 1:10, 1:12, and 1:15, respectively. The above Bac-Chl solutions were placed in a shaking incubator at $37{ }^{\circ} \mathrm{C}$ and exposed to $40 \mu \mathrm{Em}^{-2} \mathrm{~s}^{-1}$ light.

\section{$\mathrm{H}_{2}$ release from cocultured bacillus and chlorella}

Chlorella vulgaris was cultured in conical flasks containing $150 \mathrm{~mL}$ of BG11 liquid medium at $30{ }^{\circ} \mathrm{C}$ under $40 \mu \mathrm{Em}^{-2} \mathrm{~s}^{-1}$ light intensity with natural light-dark cycle. Bacillus was cultured in $500 \mathrm{~mL}$ conical flasks containing $100 \mathrm{~mL}$ LB liquid medium and placed in a shaking incubator at $37{ }^{\circ} \mathrm{C}$. Both Chlorella and Bacillus used in all experiment were in the logarithmic growth phase.

The chlorella and Bacillus solutions with the ratios of 1:10 were collected to prepare calcium alginate hydrogel beads according to our previous study. Next, we used a 0.22 $\mu \mathrm{m}$ porous polytetrafluoroethylene membrane and a transparent polyurethane film to encapsulate the hydrogel beads to manufacture Bac-Chl gel patch. Then, we placed the Bac-Chl gel in a shaking incubator at $37^{\circ} \mathrm{C}$ and exposed to $40 \mu \mathrm{Em}^{-2} \mathrm{~s}^{-1}$ light for 6,12 , 24, 48, 60 and $72 \mathrm{~h}$. The amount of $\mathrm{H}_{2}$ production of Bac-Chl gel was detected to evaluate the stability of Bac-Chl gel at different time points. The $\mathrm{H}_{2}$ concentration measurements were detected by the $\mathrm{WO}_{3}$ and gas chromatography (Agilent 7890A, USA). All the above Bac-Chl solutions were added with $100 \mathrm{mg} \mathrm{WO}_{3}$ powder respectively and cultured in a shaking incubator at $37{ }^{\circ} \mathrm{C}$ and exposed to $40 \mu \mathrm{Em}^{-2} \mathrm{~s}^{-1}$ light for $24 \mathrm{~h}$. Meanwhile, the hydrogen production of above Bac-Chl solutions with 
different ratios were detected using gas chromatograph equipped with a thermal conductivity detector.

\section{Preparation of Bac-Chl gel patch}

Sodium alginate ( $8 \%$ ) was added into the Bac-Chl solution. Subsequently, the solution was dripped into $3 \%$ calcium chloride using a $1 \mathrm{~mL}$ syringe on the magnetic stirrer to make the uniform-sized Bac-Chl gel particle. The Bac-Chl gel was encapsulated by a sterile and $0.22 \mu \mathrm{m}$ porous polytetrafluoroethylene membrane as inner lining and a transparent polyurethane film as back lining to manufacture Bac-Chl gel patch.

\section{Cell culture}

Human umbilical vein endothelial cells (HUVEC), RAW264.7 and Human Skin Fibroblasts (HSFs) were kindly obtained from Nanjing University, School of Medicine. HUVEC was incubated in RPMI1640 medium (Gibco) containing 10\% fetal bovine serum (FBS; Gibco). RAW264.7 was cultured in high-glucose Dulbecco's modified eagle medium (DMEM; Gibco) containing with 10\% heat-inactivated FBS. HSFs was cultured in DMEM supplemented with 10\% FBS. All three cell lines were cultivated in the incubator at $37^{\circ} \mathrm{C}$ and $5 \% \mathrm{CO}_{2}$.

\section{ROS scavenging ability of Bac-Chl gel}

To evaluate the ROS scavenging ability of Bac-Chl gel to promote wound healing, we used DCFH-DA and HPF fluorescent probes, respectively. RAW264.7 cells were seeded at a density of $5 \times 10^{4}$ cells per well in the lower chamber of 24 -well transwell. The control group was cultured with basal 1640 medium, the other groups were incubated with100 $\mu \mathrm{M} \mathrm{H}_{2} \mathrm{O}_{2}$ for $1 \mathrm{~h}$. Then, the $\mathrm{H}_{2} \mathrm{O}_{2}$ group was cultured with basal 1640 medium at $37^{\circ} \mathrm{C}$ and exposed to $40 \mu \mathrm{Em}^{-2} \mathrm{~s}^{-1}$ light, while Bac-Chl gel group was added Bac-Chl gel into the upper chamber of 24 -well transwell at $37^{\circ} \mathrm{C}$ and exposed to $40 \mu \mathrm{Em}^{-2} \mathrm{~s}^{-1}$ light. After $24 \mathrm{~h}$, all the above RAW264.7 cells incubated with DCFH-DA, HPF fluorescent probes at room temperature in the dark for $30 \mathrm{~min}$, were imaged under a confocal laser scanning microscope (FV1000, Olympus, Japan) and quantified using ImageJ software.

We furtherly used the Nrf2 ELISA Test Kits to evaluate the antioxidant effect of Bac-Chl gel in vitro. Briefly, according to the instructions of the Nrf2 ELISA detection 
kit, the RAW264.7 cells treated with Bac-Chl gel or not were measured the Nrf2 expression with a microplate reader.

\section{Anti-inflammatory effect of Bac-Chl gel in vitro}

In order to study the anti-inflammatory effect of the hydrogen released by Bac-Chl gel, we use LPS to induce pro-inflammatory phenotype (M1) in macrophages, and the macrophage pro-inflammatory phenotype marker CD86 and anti-inflammatory phenotype marker CD206 were observed by laser scanning confocal microscope. RAW264.7 cells were seeded in the lower chamber of a 24-well plate transwell at a density of $5 \times 10^{4}$ cells per well. The RAW264.7 cells in the control group were incubated with RPMI1640 serum-free medium, while the other cells were incubated with $100 \mu \mathrm{M}$ LPS. The Bac-Chl gel was added to the upper chamber of the transwell of the LPS + Bac-Chl gel group. All three groups of cells continue to be cultured in an incubator with light at $37^{\circ} \mathrm{C}$. After $24 \mathrm{~h}$, the RAW264.7 cells were rinsed in PBS, and then blocked with 5\% of BSA blocking solution for $1 \mathrm{~h}$. Next, RAW264.7 cells were incubated with the antibody F4/80 (Biolegend, 123109), CD86 (Biolegend, 159203), CD206 (Biolegend, 141707) at $4{ }^{\circ} \mathrm{C}$ overnight, after washed with PBS for several times, DAPI (Sigma) was used to stain nuclei for $10 \mathrm{~min}$. The sections were imaged with fluorescence microscope. and quantified using ImageJ software.

We furtherly used the IL-6/IL-10 ELISA Test Kits to evaluate the antioxidant effect of Bac-Chl gel in vitro. Briefly, according to the instructions of the IL-6/IL-10 ELISA detection kit, the RAW264.7 cells incubated with LPS and Bac-Chl gel or not were measured the IL-6/IL-10 expression with a microplate reader.

\section{Tube formation assay}

For tube formation assay, $250 \mu \mathrm{L}$ per well of thawed Matrigel (BD, USA) was added into the lower chamber of pre-cooled 24 -well plate and incubated for $1 \mathrm{~h}$ at $37^{\circ} \mathrm{C}$. Then, HUVEC cells were seeded with $33 \mathrm{mM}$ glucose medium $\left(5 \times 10^{4} /\right.$ well $)$ into the Matrigelcoated lower chamber and exposed to Bac-Chl gel into the upper chamber in the light at $1 \%$ oxygen for $12 \mathrm{~h}$. To assess the tube formation, HUVEC cells, stained by CalceinAM, were imaged under confocal laser scanning microscope and quantified using Image J software. 


\section{HSF proliferation assay}

In order to investigate the function of Bac-Chl gel on fibroblasts cultured under high glucose and hypoxia, CCK-8 assay kits were used to evaluate the viability of HSFs. HSFs were seeded with or without $33 \mathrm{mM}$ glucose medium $\left(5 \times 10^{4} /\right.$ well $)$ into the lower chamber and exposed to Bac-Chl gel into the upper chamber in the light at $1 \%$ oxygen for $24 \mathrm{~h}$. Then, the HSFs were co-incubated with CCK-8 and quantified by microplate reader.

\section{Animals}

Balb/C mice (male, about $25 \mathrm{~g}$ in weight) and high fat and sugar feed were obtained from Yangzhou University Medical Centre (Yangzhou, China). Balb/C mice were free access to abundant food and water with a group of 20. All animal operations and experimental procedures were approved in the Administration Committee of Experimental Animals in Jiangsu Province and the Ethics Committee of Nanjing University.

\section{Preparation of streptozotocin (STZ) solution}

Weigh $2.1 \mathrm{~g}$ of citric acid and add $100 \mathrm{~mL}$ of double-distilled water to prepare liquid A, and weigh $2.94 \mathrm{~g}$ of sodium citrate and add $100 \mathrm{~mL}$ of double-distilled water to prepare liquid B. At the same time, mix A and B in a certain ratio (1:1-1:1.32), and use a $\mathrm{pH}$ meter to determine the $\mathrm{pH}$ value, and adjust the $\mathrm{pH}=4.2-4.5$ to obtain the citrate buffer. Use the prepared citrate buffer solution to prepare $10 \mathrm{mg} / \mathrm{mL} \mathrm{STZ}$. The STZ preparation solution was freshly prepared just before use, and it should be used up within 30 minutes in an ice bath and protected from light.

\section{Chronic wound healing mice}

The mice were randomly divided into five groups: acute wound group (Non-DM), diabetic chronic wound group (DM), Bacillus gel patch group (DM-Bac), Chlorella gel patch group (DM-Chl), Bacillus-Chlorella gel patch group (DM- Bac+Chl). The wounds of the mice in the Non-DM and DM groups were replaced with Tegaderm membranes every 3 days, the other mice were replaced with above hydrogel patches every 3 days, respectively. All mice grow under $48 \mathrm{~h}$ of light $/ 24 \mathrm{~h}$ of darkness cycle. The area of the wound was measured and recorded with a digital camera every 3 days 
after the operation until the wound was completely healed. The wound tissues of each group were collected on the 3rd day (inflammation period), 6th day (proliferative period) and 12th day (mature period) after operation.

\section{Histology and Immunohistochemistry}

HE \& Masson staining was performed to analyze the skin tissue of granulation tissue, collagen deposition and epidermal thickness. Briefly, all skin tissues were fixed in $4 \%$ paraformaldehyde fixation solution and stained with hematoxylin-eosin or Weigert iron hematoxylin, respectively. Then, the sections were observed and photographed using a microscope connected to a digital camera. The granulation tissue and collagen fibers were observed by microscope and quantified using ImageJ software.

\section{Immunofluorescence Staining}

All tissue sections were embedded into optimal cutting temperature compound (OCT) and cryosectioned into $8 \mu \mathrm{m}$ thick frozen section in liquid nitrogen for fluorescent immunostaining. After deparaffinized by ice-cold acetone, the slice was rinsed in PBS, and then blocked with $5 \%$ of BSA blocking solution for $1 \mathrm{~h}$. To evaluate the blood vessels and inflammation, the slices were incubated with the antibody HFP (Cayman), CD31 (BioLegend, 102408), F4/80, CD86, CD206 at $4{ }^{\circ} \mathrm{C}$ overnight, after washed with PBS for several times, DAPI (Sigma) was used to stain nuclei for $10 \mathrm{~min}$. The sections were imaged with fluorescence microscope. 5 to 10 immunofluorescence images per marker were randomly collected from and quantified using ImageJ software.

\section{ELISA experiments}

The skin tissues of each group were washed with pre-cooled PBS to remove residual blood. $1 \mathrm{~mL}$ of protease inhibitor (diluted in PBS) was added per $200 \mathrm{mg}$ of tissue. The tissue was lysed at a high speed with a tissue grinder, and then the homogenate was centrifuged at $7500 \mathrm{rpm}$ for 10 minutes, and the supernatant was collected. Collect the supernatant after centrifugation. According to the Nrf2, IL-6 and IL-10 ELISA detection kit instructions, take $50 \mu \mathrm{L}$ of different concentrations of standard solution, blank solution and sample solution and add them to the microtiter plate with 5 replicate wells in each group. Then add $100 \mu \mathrm{L}$ of horseradish peroxidase (HRP)-labeled detection IL-6/IL-10 antibody to each well, seal the reaction well with a sealing film, 
and incubate it in a $37^{\circ} \mathrm{C}$ cell incubator for 60 minutes. Discard the supernatant from each well and pat dry on absorbent paper. And add $350 \mu \mathrm{L}$ washing solution to each well, wash 5 times. Add $50 \mu \mathrm{L}$ each of Substrate A and B to each well, incubate in a $37^{\circ} \mathrm{C}$ incubator in the dark for 15 minutes. Add $50 \mu \mathrm{L}$ of stop reaction solution to each well, and measure the OD value of each well with a microplate reader at $450 \mathrm{~nm}$ wavelength. The concentration-absorbance standard curve is made according to the absorbance values of different concentrations, and the concentration of each sample well is calculated according to the standard curve to obtain the expression level of Nrf2, IL-6/IL-10 of the cells and skin tissues, respectively.

\section{Statistical analysis}

Statistical significance was determined using unpaired and paired two-sided Student's t-tests as indicated; data presented as single measurements with bars as group means \pm s.d. For histochemical analysis, a one-way ANOVA with Holm-Sidak's multiple comparisons was used to determine statistical significance; data are mean \pm s.d.; $p<$ 0.05 was considered significant. 


\section{Supplementary Figures}
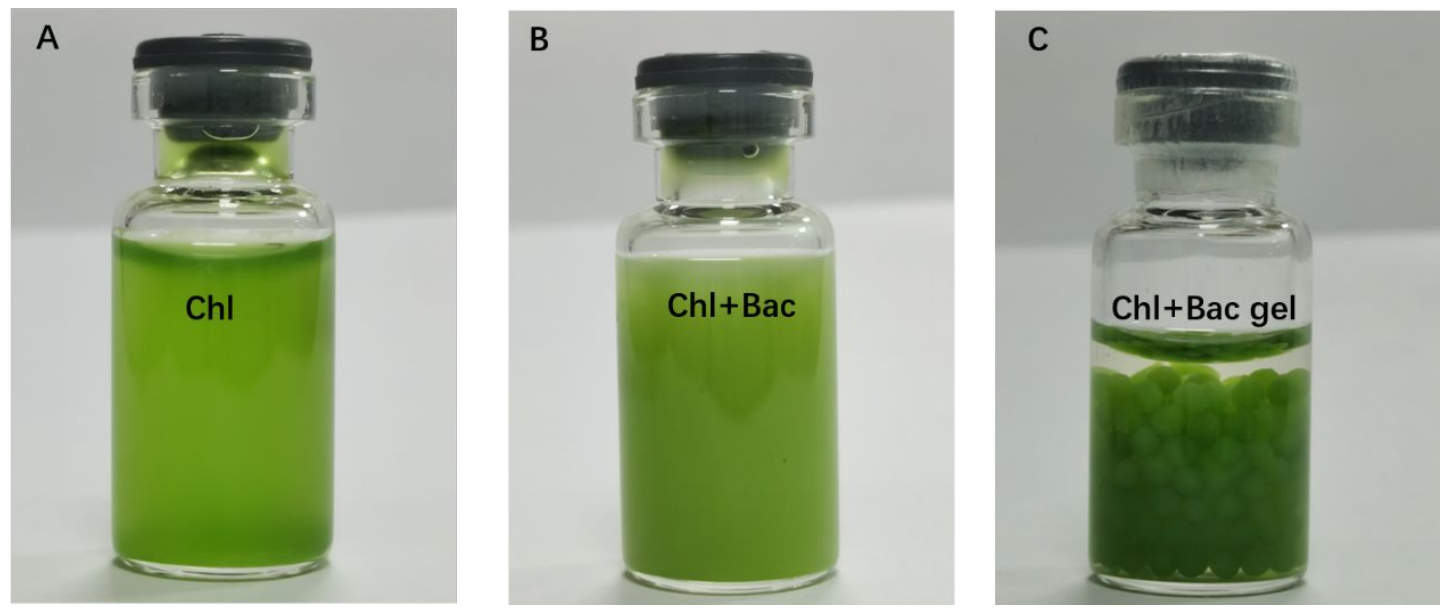

Figure S1. Photos of chlorella solution (A), chlorella and Bacillus solution (B), chlorella and Bacillus gel (C).

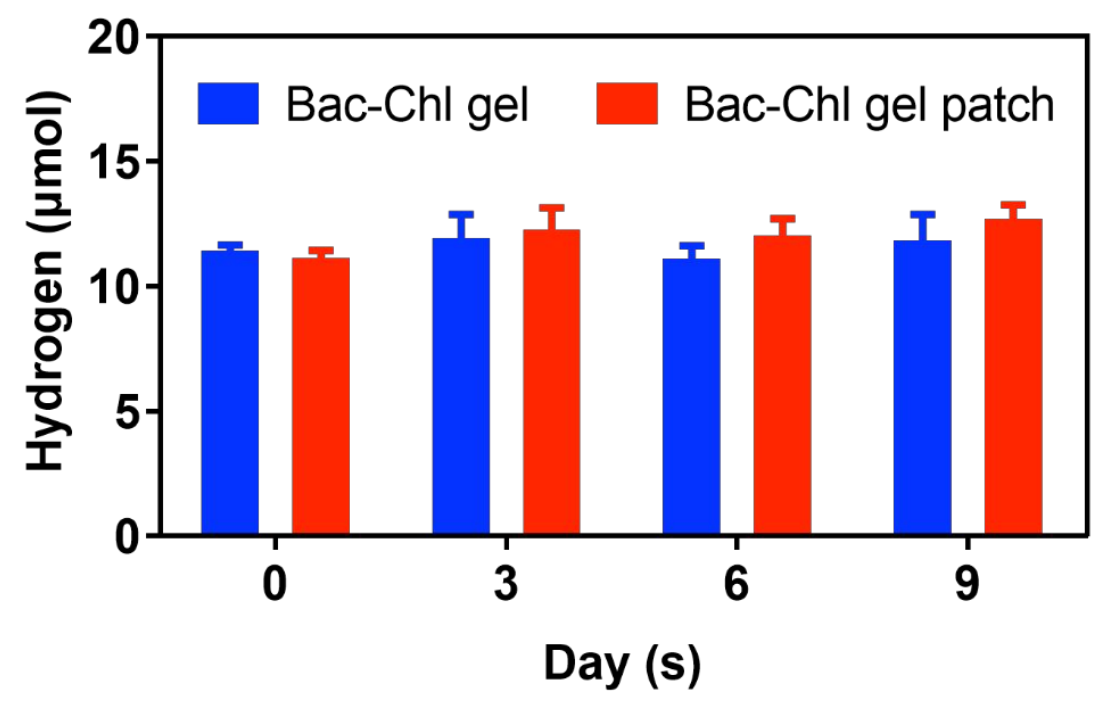

Figure S2. Comparison of releasing hydrogen during the storage of Bac-Chl gel patch stored at $4{ }^{\circ} \mathrm{C}$ under dark conditions for 3, 6, 9 days and then cultured under light conditions for $60 \mathrm{~h}$. 


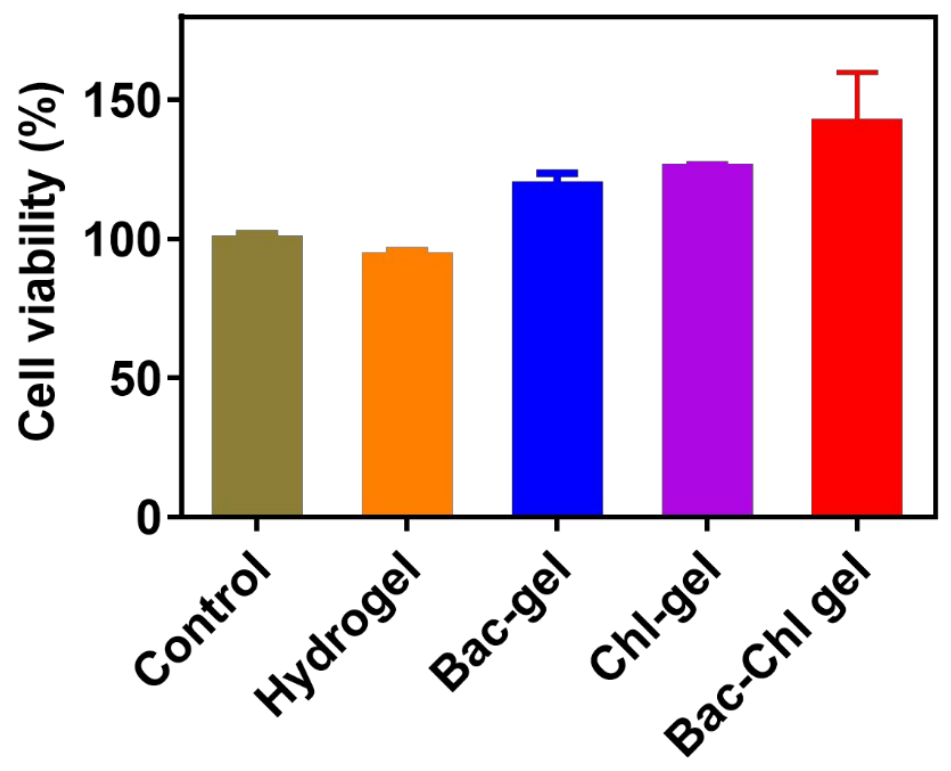

Figure S3. HUVEC cell viability for different groups.

A

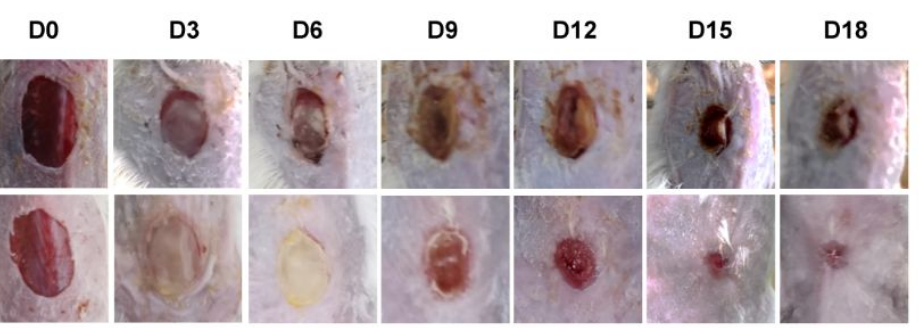

B

C
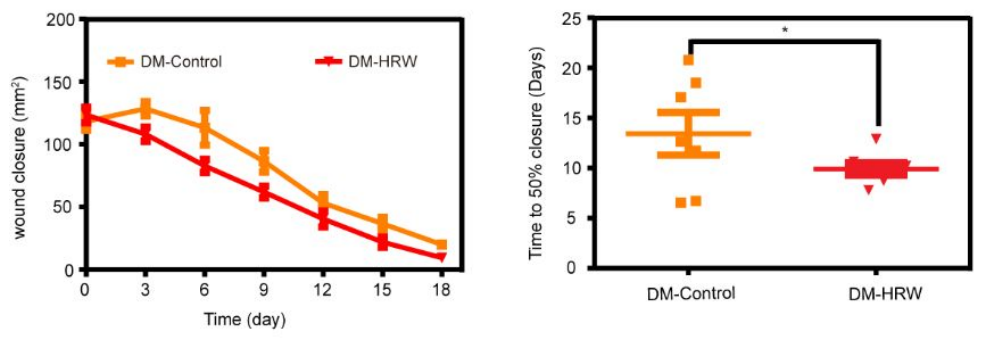

Figure S4. The promoting effects on diabetic wound healing of HRW. (A) Representative images of the wound area with or without HRW on day $0,3,6,9,12,15$ and 18 after operation $(n=6)$. (B) Fractions of wounds healed by HRW treatments at days $3,6,9,12,15$ and $18(\mathrm{n}=6)$. (C) The curve of wound healing $(\mathrm{n}=6)$. Significantly different (one-way ANOVA): ${ }^{*} p<0.05$. 

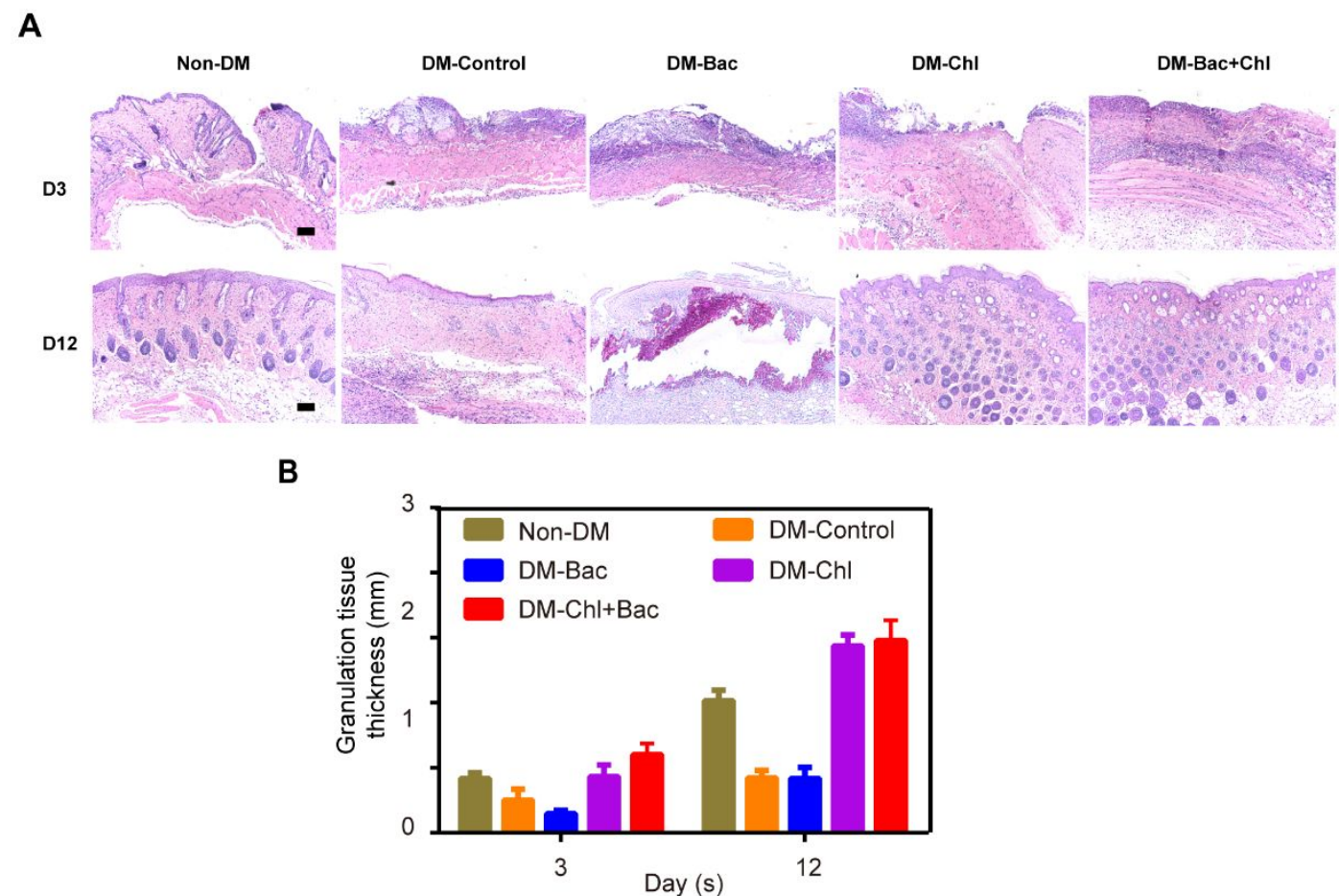

Figure S5. H\&E staining of the wound tissue at inflammatory and re-epithelialization stages in different groups. (A) H\&E staining of the wound area reflected the regenerated skin in different groups at day 3 and $12(\mathrm{n}=3)$. Scale bars, $100 \mu \mathrm{m}$. (B) Quantification of the granulation tissue in different groups at day 3 and $6(n=3)$. Significantly different (one-way ANOVA).
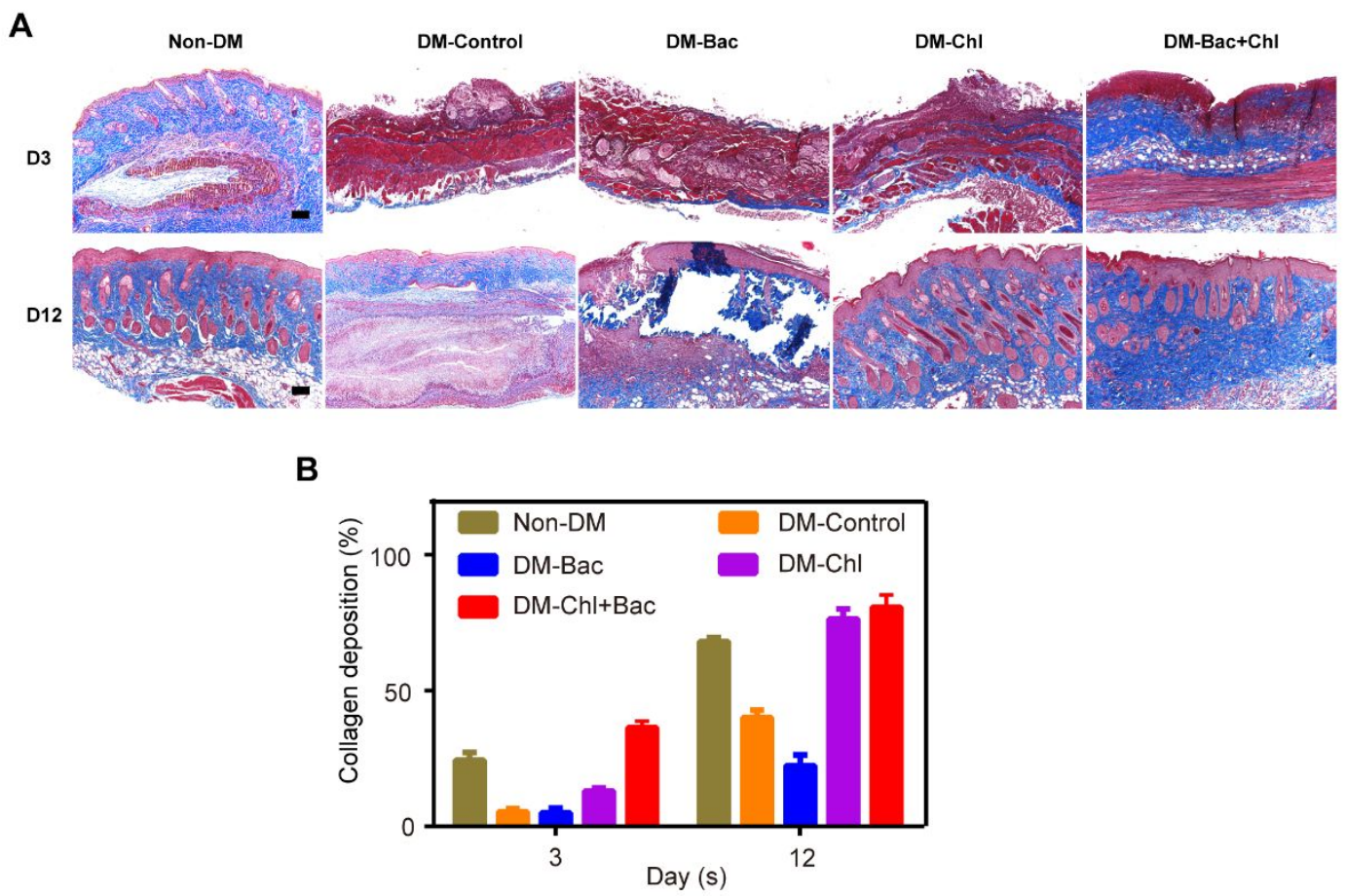

Figure S6. Masson staining of the wound tissue at the inflammatory and re-epithelialization stages 
in different groups. (A) Masson staining of the wound area reflected the regenerated skin in different groups at day 3 and $12(n=3)$. Scale bars, $100 \mu \mathrm{m}$. (B) Quantification of the collagen deposition in different groups at day 3 and $6(\mathrm{n}=3)$. Significantly different (one-way ANOVA). 